Original Article

\title{
Population distribution and habitat analysis of Rufous treepie (Dendrocitta vagabunda) in Abbottabad, Pakistan
}

\author{
Distribuição da população e análise de habitat de Rufous treepie (Dendrocitta \\ vagabunda) em Abbottabad, Paquistão
}

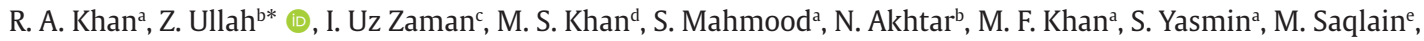 \\ A. Ur Rehmanª, N. Aksara, S. N. Khana and S. S. Hussain ${ }^{\mathrm{a}}$ \\ aHazara University Mansehra, Department of Zoology, Khyber Pakhtunkhwa, Pakistan

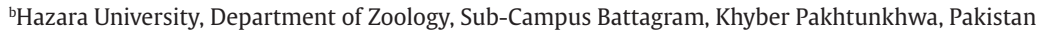 \\ 'Khyber Pakhtunkhwa Wildlife Department, Pakistan \\ dUniversity of Swabi, Department of Zoology, Khyber Pakhtunkhwa, Pakistan \\ eHorizon Degree College Chakwal, Department of Zoology, Pakistan
}

\begin{abstract}
The Rufous treepie (Dendrocitta vagabunda) belongs to family corvidae, order Passeriformes which includes about 100 species. The current study was conducted to gather information about the Population distribution and habitat analysis of $D$. vagabunda at District Abbottabad, Pakistan. The data were collected on monthly basis both morning and evening times (2018-2019). "The "Point count Method" was used for population estimation and "Quadrates Method" for habitat analysis of study area. The result shows an average month-wise population density of $D$. vagabunda was maximum at Jhangra $0.14 \pm 0.039 /$ ha, whereas minimum at Havelian $0.11 \pm 0.022 /$ ha. There was no significant difference ( $p>0.05$ ) among monthly population densities of $D$. vagabunda, however, a significant difference $(p<0.05)$ was found between morning and evening times population of the specie. The present study revealed that importance value index (IVI) of plants species at Sherwan, Bakot, Havelian, Langra and Jhangra were 59.6 $\pm 12.6,50.1 \pm 6.9,53.4 \pm 6.3,66.8 \pm 10$ and $60.1 \pm 7.7$. Likewise, the frequency of shrubs at Sherwan, Bakot, Havelian, Langra and Jhangra were $33.3 \pm 4.2,45 \pm 9.4,46.7 \pm 8.2,55.6 \pm 22.2$ and $37.5 \pm 8.5$. Similarly, the frequency of herbs at Sherwan, Bakot, Havelian, Langra and Jhangra were 40.4 $\pm 6.0,37.5 \pm 5.6,53.3 \pm 7.4,48.5 \pm 5.2$ and $46.9 \pm 7.4$ respectively. Our results show the study area as suitable habitat for $D$. vagabunda.
\end{abstract}

Keywords: population, quadrate method, habitat analysis, Abbottabad, Dendrocitta vagabunda.

\begin{abstract}
Resumo
A trepadeira Rufous (Dendrocitta vagabunda) pertence à família corvidae, ordem Passeriformes que inclui cerca de 100 espécies. $O$ estudo atual foi realizado para reunir informações sobre a distribuição da população e análise do habitat de $D$. vagabunda no distrito de Abbottabad, Paquistão. Os dados foram coletados mensalmente pela manhã e à noite (2018-2019). O "método de contagem de pontos" foi usado para estimativa da população e o "método dos quadrados" para análise de habitat da área de estudo. O resultado mostra que uma densidade populacional média mensal de $D$. vagabunda foi máxima em Jhangra 0,14 $\pm 0,039$ / ha, enquanto a mínima em Havelian 0,11 $\pm 0,022 /$ ha. Não houve diferença significativa ( $p>0,05$ ) entre as densidades populacionais mensais de $D$. vagabunda, entretanto foi encontrada diferença significativa $(\mathrm{p}<0,05)$ entre os períodos matutino e noturno da população da espécie. 0 presente estudo revelou que o índice de valor de importância (IVI) das espécies de plantas em Sherwan, Bakot, Havelian, Langra e Jhangra foi de: $59,6 \pm 12,6,50,1 \pm 6,9,53,4 \pm 6,3,66,8 \pm 10$ e 60,1 $\pm 7,7$. Da mesma forma, a frequência de arbustos em Sherwan, Bakot, Havelian, Langra e Jhangra foi de: 33,3 $\pm 4,2,45 \pm 9,4,46,7 \pm 8,2$, $55,6 \pm 22,2$ e $37,5 \pm 8,5$. Da mesma forma, a frequência de ervas em Sherwan, Bakot, Havelian, Langra e Jhangra foi: $40,4 \pm 6,0,37,5 \pm 5,6,53,3 \pm 7,4,48,5 \pm 5,2$ e 46,9 $\pm 7,4$, respectivamente. Nossos resultados mostram a área de estudo como habitat adequado para $D$. vagabunda.
\end{abstract}

Palavras-chave: população, método dos quadrados, análise de habitat, Abbottabad, Dendrocitta vagabunda.

*e-mail: zaibullah_zoology@hu.edu.pk

Received: December 27, 2020 - Accepted: February 10, 2021 


\section{Introduction}

Birds occupy a wide range of ecological positions (Sekercioglu, 2006; Mirza and Wasiq, 2012). Globally, Rufous treepie (Dendrocitta vagabunda) is distributed in Burma, India, Bangladesh, Bhutan, Cambodia, Myanmar, Nepal, Thailand, Vietnam and Pakistan (BirdLife International, 2013). The specie is widely distributed in whole of Punjab, Sindh, in South-eastern corner of Baluchistan including low hills of Azad Kashmir, Kohat and Peshawar valley and common in Islamabad, Margalla hills and Punjab and further spread towards Hazara (KP) (Mirza and Wasiq, 2012; Hornman, 2007; Jadoon et al., 2019; Ullah et al., 2020).

Density and abundance are the essential ecological information required for population ecology and scope of ecology covers distribution of organism and its abundance (Buckland et al., 2001; Stephens et al., 2019).D. vagabunda is fairly wide spreads throughout Indus plains and foot hills up to $2300 \mathrm{~m}$ and prefers tree or bush cover and occupies altitudinal range of $1450-1700 \mathrm{~m}$ in Bhowali, while its altitudinal range lies between $350-500 \mathrm{~m}$ in Haldwani along with its status is recorded as residential species in Nainital district under conservation status of Indian wildlife protection Act (Grimmett et al., 2008; Joshi et al., 2012).

The $D$. vagabunda is arboreal bird of the teak forests, but seems at homes, in open scrub jungle, gardens, cultivated trees and scrub forest areas, not in desert without trees and prefers shrubby habitat (Sidhu et al., 2010; Mirza and Wasiq, 2012). The D. vagabunda occupied in whole of sub-tropical forest and tropical evergreen forest, and found abundant in all habitats, as a residential and winter visitor spp in Muzaffrabad city in Azad Jammu and Kashmir in Pakistan, also found in coniferous forest, mixed deciduous forests of scrubby areas in Chenani in lesser Himalayas in Jammu and Kashmir, India (Shahabuddin et al., 2006; Thakur et al., 2010; Singh et al., 2013).

\section{Materials and Methods}

\subsection{Study area}

The present study was conducted in District Abbottabad, positioned at an altitude of 1,225 meter, lies between $33^{\circ} 50^{\prime}$ and $34^{\circ} 23^{\prime}$ North latitude and $73^{\circ} 35^{\prime}$ and $73^{\circ} 31^{\prime}$ East longitude (Figure 1 ) with $1,967 \mathrm{~km}^{2}$ area (Ali et al., 2017). Most of the area of District is comprised of mountainous topography and the average elevation of mountains ranges from $2500 \mathrm{~m}$ to $2700 \mathrm{~m}$ (IUCN Pakistan, 2004; Ullah et al., 2020). The aim of study was to determine the population density, distribution and habitat preference of $D$. vagabunda including Sherwan and Bakot (hilly areas), Havelian, Jhangra and Langra (plane areas) with the total area of 6942 hectares.

\subsection{Methods}

\subsubsection{Population estimation}

Field surveys were conducted (2018-2019). The "Point Count Method" was used in hilly area (Sherwan and Bakot) and plane area (Havelian, Langra and Jhangra). Preliminary surveys were conducted to allow covering a wider range of the potential habitats of $D$. vagabunda. In point count method "Vantage points" were selected of fixed radius $\left(50 \mathrm{~m}^{2}\right)$ and duration of time (15 minutes) for population estimation both at morning and evening time (Rolando et al., 2007).

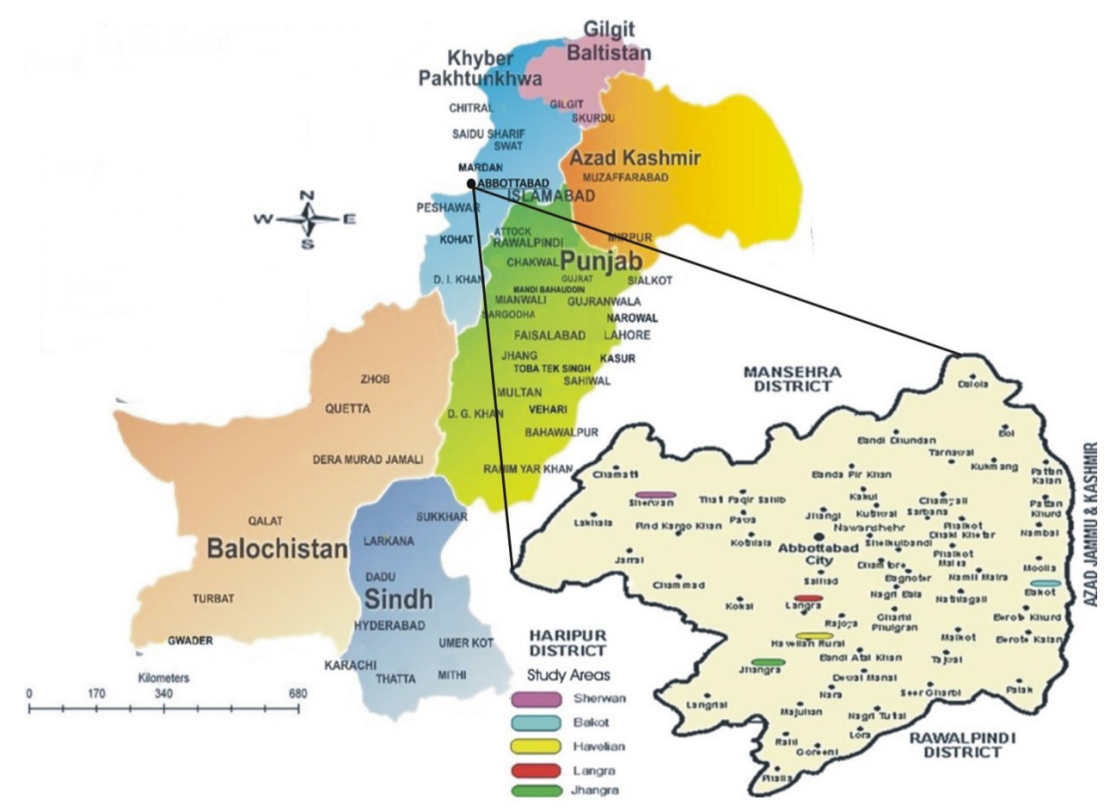

Figure 1. Map of the study area indicate District Abbottabad. 


\subsubsection{Estimation of population density}

Population density estimation was carried through sighting observations to assess the accuracy of perpendicular measurement by Visual Encounter Method (VEM) (Ralph et al., 1995). Data recording was done by thoroughly surveys on monthly basis for the whole study period. The measurements were recorded on flushing the bird (Buckland et al., 2008).

Population density of $D$. vagabunda is represented by Formula 1.

$$
\text { Density }=\frac{\text { Number of birds }}{\partial \mathrm{r}^{2}}
$$

where, $\mathrm{r}=$ radius of circle $=50 \mathrm{~m}^{2}$.

Total population $=$ No of D. vagabunda in all sites $\times$

Total area of study sites

\subsection{Habitat analysis}

In order to analyze the preferred habitat used by D. vagabunda, all major plant species were collected, identified and preserved in the form of herbarium sheets. Vegetation sampling was carried out by using "Quadrates Method" as described by (Sigdel, 2008). Among the vegetation, trees were examined by using quadrates of $(10 \times 10 \mathrm{~m})$, shrubs $(5 \times 5 \mathrm{~m})$ and herbs $(1 \times 1 \mathrm{~m})$ (Ali et al., 2018).

\subsubsection{Vegetation analysis}

The collected data were used to calculate species richness, density, relative density, frequency and relative frequency of trees, shrubs and herbs in the study area by using the following Formulas 3 to 7.

$$
\begin{aligned}
& \text { Density of species }=\frac{\text { Total number of individuals of species A }}{\text { Total number of areas surveyed } \times \text { Area of plot }} \\
& \text { Relative density of species }=\frac{\text { Total number of individuals of species A }}{\text { Total number of individuals of all species }} \\
& \text { Frequency of species }=\frac{\text { Number of plots in which species A occurs } \times 100}{\text { Total number of plot samples }} \\
& \text { Relative Frequency of species }=\frac{\text { Frequency value of species A } \times 100}{\text { Total frequency value of all species }} \\
& \text { Relative dominance of species }=\frac{\text { Total basal area of species A } \times 100}{\text { Total basal area of all species }}
\end{aligned}
$$

Importance Value Index (IVI) was calculated as Formula 8 (Aryal et al., 2010):

$$
\begin{aligned}
& I V I=\text { Relative density }+ \text { Relative frequency }+ \\
& \text { Relative dominance }
\end{aligned}
$$

\subsection{Statistical analysis}

Analysis of Variance (ANOVA) was applied for analysis of data regarding in different habitats for month-wise population densities of $D$. vagabunda. Student's paired "t-test" was applied for comparison between morning and evening time's population densities.

\section{Results}

\subsection{Month-wise population estimation}

Month-wise population density of $D$. vagabunda was estimated at five sites of the study area. Average month-wise population density of the $D$. vagabunda was maximum in Jhangra $(0.14 \pm 0.039 /$ ha $)$ while minimum in Havelian $(0.11 \pm 0.022 /$ ha) (Table 1$)$ (Figure 2).

\subsection{Fluctuation of D. vagabunda population}

The morning and evening population density of D. vagabunda was considerably higher during morning as compared to that in evening time and this trend was consistently observed during the entire study period. Population density of $D$. vagabunda during morning was greater $0.038 \pm 0.0039 /$ ha, than that of evening time $(0.032 \pm 0.0019 /$ ha $)$ in Sherwan. Similarly, its population density was maximum $(0.031 \pm 0.0032 / \mathrm{ha})$, at morning and minimum $(0.028 \pm 0.0032 / \mathrm{ha})$ at evening time in Bakot. The population density at morning and evening was $0.037 \pm 0.0042$ /ha and $0.036 \pm 0.0025 /$ ha, in Havelian respectively. In Langra, the maximum population density was $0.042 \pm 0.0047 / \mathrm{ha}$, at morning and higher $0.033 \pm 0.0054 /$ ha, at evening time (Figure 3 ). The population density was found $0.037 \pm 0.0038 /$ ha, during morning and $(0.035 \pm 0.0026 /$ ha $)$ at evening in Jhangra (Table 2) (Figure 4).

\subsection{Habitat analysis}

The presence of abundant trees, shrubs and herbs are essential for the survival of $D$. vagabunda at any area. The importance value index for trees, frequencies of shrubs and herbs indicates the relative population of $D$. vagabunda might survive in this area.

The major tree species for D. vagabunda at Sherwan site having dominant IVI 95.14 of Zanthoxylum armatum with co-dominant IVI 126.85 was recorded for Grewia optiva, whereas minimum IVI was observed for Melia azedarach and Morus alba 31.68. Similarly, the frequency of shrubs $33.3 \pm 4.2$ and herbs was $40.4 \pm 6.0$ (Table 3, 4 and 5).

At Bakot, the dominant tree species having IVI 62.77 of Ailanthus altissima with co-dominant IVI was recorded for Acacia modesta 93.87, and minimum IVI was observed for Melia azedarach 31.26. Therefore the frequency of shrubs and herbs at this site was $45 \pm 9.4$ and $37.5 \pm 5.6$ respectively (Table 6, 7 and 8).

The dominant tree species at Havelian having IVI 82.17 of Dalbergia sissoo and co-dominant IVI was observed for Broussonetia papyrifera 82.29, while minimum IVI was recorded for Melia azedarach 41.04. Similarly, the frequency of shrubs $46.7 \pm 8.2$ and ground cover was 53.3 \pm 7.4 (Table 9, 10 and 11).

At Langra, among tree species the dominant IVI 89.06 of Ficus palmata with co-dominant IVI was recorded for Broussonetia papyrifera 89.44 , and minimum IVI was observed for Morus alba and Eucalyptus camaldulensis 44.46. The frequency of shrubs and herbs at this site was

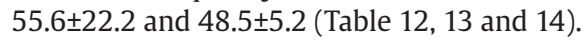

The major tree species having dominant IVI 90.16 of Olea ferruginea Royle with co-dominant IVI was recorded for Broussonetia papyrifera 90.34, whereas minimum IVI was observed for Ficus palmata 30.04 in Jhangra. Similarly frequency of shrubs $37.5 \pm 8.5$ and herbs was $46.9 \pm 7.4$ respectively (Table 15,16 and 17 ). 


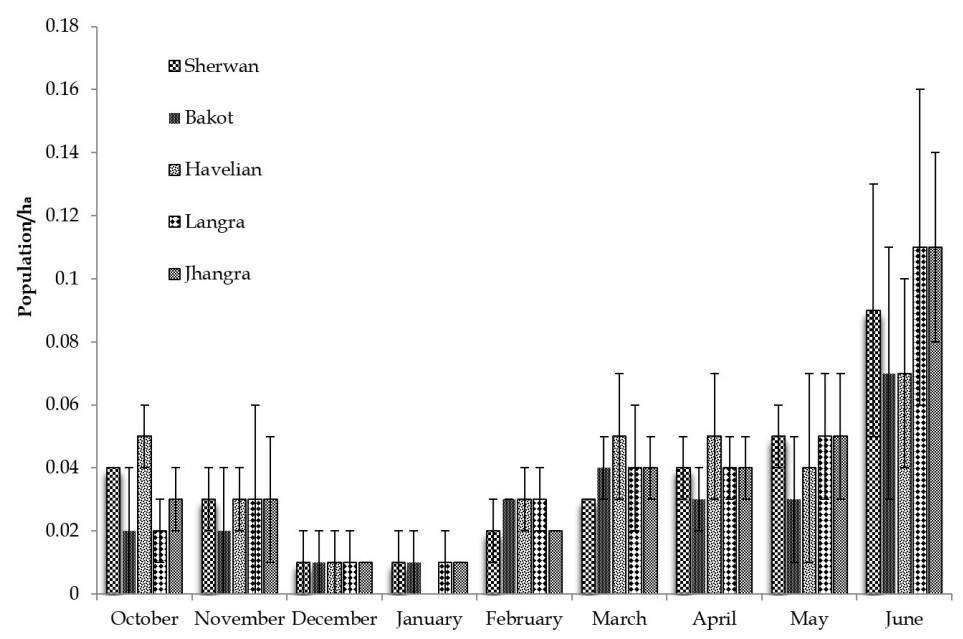

Figure 2. Overall month-wise population densities of D. vagabunda from five selected sites during the study period.

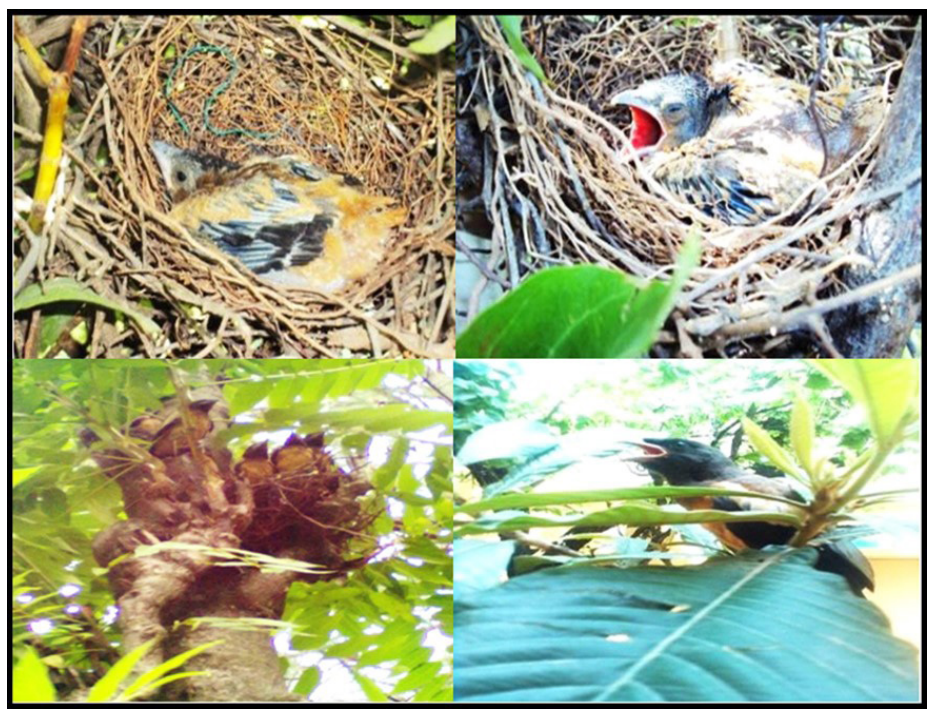

Figure 3. Snapshots of $D$. vagabunda captured during field survey of population estimation.

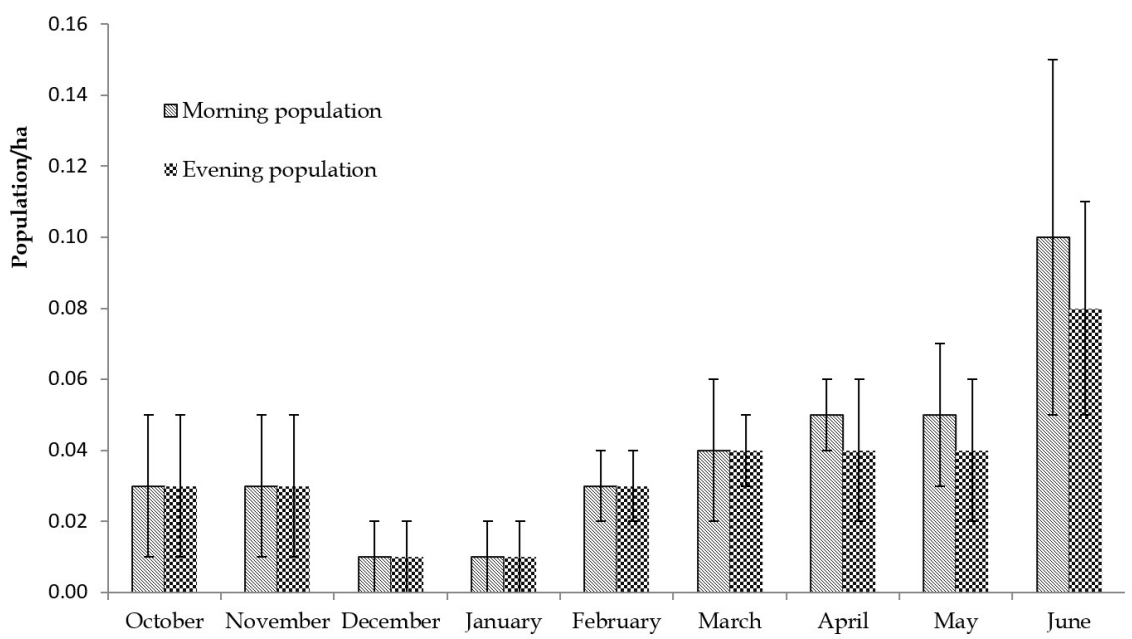

Figure 4. Overall morning and evening population densities/ha of Dendrocitta vagabunda during the study period in all selected study sites. 
Table 1. Total month-wise Population density of D. vagabunda in selected sites of district Abbottabad.

\begin{tabular}{ccccc}
\hline Study sites & Area in ha & $\begin{array}{c}\text { Population } \\
\text { density/ha }\end{array}$ & Total population & P-value \\
\hline Sherwan & 1376 & $0.13 \pm 0.035$ & 47 \\
Bakot & 1422 & $0.13 \pm 0.029$ & 51 \\
Havelian & 1613 & $0.11 \pm 0.022$ & 61 \\
Langra & 1706 & $0.11 \pm 0.033$ & 57 & 31 \\
Jhangra & 825 & $0.14 \pm 0.039$ & $\mathbf{2 4 7}$ & $(\boldsymbol{p}>\mathbf{0 . 0 5})$ \\
Total & $\mathbf{1 3 8 8 . 4 0 \pm 3 4 2 . 7 8}$ & $\mathbf{0 . 1 2} \pm \mathbf{0 . 0 3 1}$ & & \\
\hline
\end{tabular}

Table 2. Average Population Density of D. vagabunda at selected study sites at morning and evening time in District Abbottabad.

\begin{tabular}{|c|c|c|c|c|c|c|c|}
\hline Tehsil & Study sites & Area in ha & Day Time & $\begin{array}{l}\text { Population } \\
\text { Density/ha }\end{array}$ & $\begin{array}{c}\text { Total } \\
\text { population }\end{array}$ & $\begin{array}{l}P(T<=t) \\
\text { one-tail }\end{array}$ & $\begin{array}{l}P(T<=t) \\
\text { two-tail }\end{array}$ \\
\hline \multirow[t]{4}{*}{ Abbottabad } & Sherwan & 1376 & $\begin{array}{l}\text { Morning } \\
\text { time }\end{array}$ & $0.038 \pm 0.0039$ & 52 & & \\
\hline & & & $\begin{array}{l}\text { Evening } \\
\text { time }\end{array}$ & $0.032 \pm 0.0019$ & 44 & & \\
\hline & Bakot & 1422 & $\begin{array}{l}\text { Morning } \\
\text { time }\end{array}$ & $0.031 \pm 0.0032$ & 44 & & \\
\hline & & & $\begin{array}{l}\text { Evening } \\
\text { time }\end{array}$ & $0.028 \pm 0.0032$ & 40 & & \\
\hline \multirow[t]{6}{*}{ Havelian } & Havelian & 1613 & $\begin{array}{l}\text { Morning } \\
\text { time }\end{array}$ & $0.037 \pm 0.0042$ & 60 & & \\
\hline & & & $\begin{array}{c}\text { Evening } \\
\text { time }\end{array}$ & $0.036 \pm 0.0025$ & 58 & & \\
\hline & Langra & 1706 & $\begin{array}{l}\text { Morning } \\
\text { time }\end{array}$ & $0.042 \pm 0.0047$ & 72 & & \\
\hline & & & $\begin{array}{l}\text { Evening } \\
\text { time }\end{array}$ & $0.033 \pm 0.0054$ & 56 & & \\
\hline & Jhangra & 825 & $\begin{array}{l}\text { Morning } \\
\text { time }\end{array}$ & $0.037 \pm 0.0038$ & 31 & & \\
\hline & & & $\begin{array}{c}\text { Evening } \\
\text { time }\end{array}$ & $0.035 \pm 0.0026$ & 29 & & \\
\hline Total & & 6942 & & & 486 & & \\
\hline \multirow[t]{3}{*}{ Mean $\pm S . D$} & & $1388.40 \pm 342.78$ & $\begin{array}{l}\text { Morning } \\
\text { time }\end{array}$ & & $51.80 \pm 15.56$ & & \\
\hline & & & $\begin{array}{l}\text { Evening } \\
\text { time }\end{array}$ & & $45.40 \pm 11.95$ & & \\
\hline & & & & & & 0.004449923 & 0.008899846 \\
\hline
\end{tabular}

Total population $=$ No of $D$. vagabunda in all transect $\times$ Total area of study sites. Total selected study area of Abbottabad $=6942$ hectares. The average collective population at morning and evening times was estimated to be 486 .

Table 3. Density, frequency and IVI of Trees species at Sherwan site in district Abbottabad.

\begin{tabular}{|c|c|c|c|c|c|c|c|}
\hline $\begin{array}{l}\text { Sr. } \\
\text { No }\end{array}$ & Scientific Name & $\begin{array}{c}\text { Density/ } 10 \\
\mathrm{~m}^{2}\end{array}$ & $\begin{array}{l}\text { Relative } \\
\text { Density }\end{array}$ & Frequency & $\begin{array}{l}\text { Relative } \\
\text { Frequency }\end{array}$ & $\begin{array}{c}\text { Relative } \\
\text { Dominance }\end{array}$ & IVI \\
\hline 1 & Celtis caucasica & 0.05 & 0.04 & 25 & 6.66 & 25 & 31.7 \\
\hline 2 & Ficus palmate & 0.15 & 0.12 & 50 & 13.3 & 50 & 63.42 \\
\hline 3 & Grewia optiva & 0.22 & 0.19 & 100 & 26.66 & 100 & 126.85 \\
\hline 4 & Melia azedarach & 0.02 & 0.02 & 25 & 6.66 & 25 & 31.68 \\
\hline 5 & Morus alba & 0.02 & 0.02 & 25 & 6.66 & 25 & 31.68 \\
\hline 6 & Olea ferruginea Royle & 0.07 & 0.63 & 25 & 6.66 & 25 & 32.29 \\
\hline 7 & Pinus roxburghii & 0.45 & 0.38 & 50 & 13.3 & 50 & 63.68 \\
\hline \multirow[t]{2}{*}{8} & $\begin{array}{l}\text { Zanthoxylum } \\
\text { armatum }\end{array}$ & 0.17 & 0.14 & 75 & 20 & 75 & 95.14 \\
\hline & Mean $\pm S . E$ & $0.1 \pm 0.05$ & $0.2 \pm 0.08$ & $46.9 \pm 10$ & $12.5 \pm 2.7$ & $46.9 \pm 10$ & $59.6 \pm 12.6$ \\
\hline
\end{tabular}

S.E is a measure of the statistical accuracy of an estimate. 
Table 4. Density and frequency of Shrubs species at Sherwan site in district Abbottabad.

\begin{tabular}{cccccc}
\hline Sr. No & Scientific Name & Density $/ \mathbf{5} \mathbf{~ m}^{\mathbf{2}}$ & Relative Density & Frequency & Relative Frequency \\
\hline $\mathbf{9}$ & Barleria cristata & 0.2 & 0.04 & 25 & 7.69 \\
$\mathbf{1 0}$ & Berberis vulgaris & 0.3 & 0.07 & 25 & 7.69 \\
$\mathbf{1 1}$ & Elaeagnus umbellate & 0.05 & 0.01 & 25 & 7.69 \\
$\mathbf{1 2}$ & Daphne mucronata & 0.2 & 0.04 & 25 & 7.69 \\
$\mathbf{1 3}$ & Dicliptera clinopodia & 0.4 & 0.09 & 25 & 7.69 \\
$\mathbf{1 4}$ & Dodonaea viscosa & 0.2 & 0.04 & 50 & 15.38 \\
$\mathbf{1 5}$ & Malvastrum coromandelianm & 1.4 & 0.34 & 50 & 15.38 \\
$\mathbf{1 6}$ & Punica granatum & 0.5 & 0.12 & 25 & 15.38 \\
$\mathbf{1 7}$ & Rumex hastatus & 0.85 & 0.2 & 50 & $\mathbf{3 3 . 3 \pm 4 . 2}$ \\
\end{tabular}

Table 5. Density and frequency of Herbs species at Sherwan site in district Abbottabad.

\begin{tabular}{|c|c|c|c|c|c|}
\hline Sr. No & Scientific Name & Density/ $\mathbf{m}^{2}$ & Relative Density & Frequency & $\begin{array}{c}\text { Relative } \\
\text { Frequency }\end{array}$ \\
\hline 18 & Alternanthera pungens & 1.75 & 0.05 & 25 & 4.76 \\
\hline 19 & Amaranthus spinosus & 1.25 & 0.03 & 25 & 4.76 \\
\hline 20 & Clematis graveolens & 0.75 & 0.02 & 50 & 9.52 \\
\hline 21 & Conyza canadensis & 2.5 & 0.07 & 25 & 4.76 \\
\hline 22 & Dichanthium annulatum & 3.75 & 0.11 & 25 & 4.76 \\
\hline 23 & Eleusine indica & 5.5 & 0.16 & 50 & 9.52 \\
\hline 24 & Eragrostis curvula & 3.75 & 0.11 & 50 & 9.52 \\
\hline 25 & Euphorbia hirta & 1.75 & 0.05 & 50 & 9.52 \\
\hline 26 & Geranium dalmaticum & 4.5 & 0.13 & 100 & 19.04 \\
\hline 27 & Oxalis corniculata & 3.25 & 0.09 & 50 & 9.52 \\
\hline 28 & Pennisetum hohenackeri & 3 & 0.09 & 25 & 4.76 \\
\hline 29 & Plantago lanceolata & 0.25 & 0.007 & 25 & 4.76 \\
\hline \multirow[t]{2}{*}{30} & Tagetes minuta & 1 & 0.03 & 25 & 4.76 \\
\hline & Mean $\pm S . E$ & $2.5 \pm 0.44$ & $0.1 \pm 0.01$ & $40.4 \pm 6.0$ & $7.7 \pm 1.1$ \\
\hline
\end{tabular}

Table 6. Density, frequency and IVI of Trees species at Bakot site in district Abbottabad.

\begin{tabular}{|c|c|c|c|c|c|c|c|}
\hline Sr. No & Scientific Name & $\begin{array}{l}\text { Density/ } \\
10 \mathrm{~m}^{2}\end{array}$ & $\begin{array}{l}\text { Relative } \\
\text { Density }\end{array}$ & Frequency & $\begin{array}{l}\text { Relative } \\
\text { Frequency }\end{array}$ & $\begin{array}{c}\text { Relative } \\
\text { Dominance }\end{array}$ & IVI \\
\hline 31 & Acacia modesta & 0.22 & 0.12 & 75 & 18.75 & 75 & 93.87 \\
\hline 32 & Ailanthus altissima & 0.5 & 0.27 & 50 & 12.5 & 50 & 62.77 \\
\hline 33 & Broussonetia papyrifera & 0.07 & 0.04 & 25 & 6.25 & 25 & 31.29 \\
\hline 34 & Ficus palmate & 0.3 & 0.16 & 50 & 12.5 & 50 & 62.66 \\
\hline 35 & Melia azedarach & 0.02 & 0.01 & 25 & 6.25 & 25 & 31.26 \\
\hline 36 & Morus alba & 0.1 & 0.05 & 50 & 12.5 & 50 & 62.55 \\
\hline 37 & Pinus roxburghii & 0.05 & 0.02 & 25 & 6.25 & 25 & 31.27 \\
\hline 38 & $\begin{array}{l}\text { Populus grandidentata } \\
\text { Michx }\end{array}$ & 0.07 & 0.04 & 25 & 6.25 & 25 & 31.29 \\
\hline 39 & Robinia pseudoacacia & 0.4 & 0.21 & 50 & 12.5 & 50 & 62.71 \\
\hline \multirow[t]{2}{*}{40} & Vitex pinnata & 0.07 & 0.04 & 25 & 6.25 & 25 & 31.29 \\
\hline & Mean $\pm S . E$ & $0.2 \pm 0.05$ & $0.1 \pm 0.03$ & $40 \pm 5.5$ & $10 \pm 1.4$ & $40 \pm 5.5$ & $50.1 \pm 6.9$ \\
\hline
\end{tabular}


Table 7. Density and frequency of Shrubs species at Bakot site in district Abbottabad.

\begin{tabular}{|c|c|c|c|c|c|}
\hline Sr. No & Scientific Name & Density/5 m² & Relative Density & Frequency & $\begin{array}{l}\text { Relative } \\
\text { Frequency }\end{array}$ \\
\hline 41 & Barleria cristata & 0.5 & 0.19 & 50 & 22.22 \\
\hline 42 & $\begin{array}{c}\text { Malvastrum } \\
\text { coromandelianum }\end{array}$ & 1.05 & 0.4 & 75 & 33.33 \\
\hline 43 & $\begin{array}{l}\text { Dicliptera } \\
\text { clinopodia }\end{array}$ & 0.25 & 0.09 & 25 & 11.11 \\
\hline 44 & $\begin{array}{c}\text { Solanum } \\
\text { pseudocapsicum }\end{array}$ & 0.2 & 0.07 & 25 & 11.11 \\
\hline \multirow[t]{2}{*}{45} & Dodonaea viscosa & 0.6 & 0.23 & 50 & 22.22 \\
\hline & Mean士S.E & $0.5 \pm 0.15$ & $0.2 \pm 0.06$ & $45 \pm 9.4$ & $20 \pm 4.2$ \\
\hline
\end{tabular}

Table 8. Density and frequency of Herbs species at Bakot site in district Abbottabad.

\begin{tabular}{|c|c|c|c|c|c|}
\hline Sr. No & Scientific Name & Density/ $\mathbf{m}^{2}$ & Relative Density & Frequency & $\begin{array}{c}\text { Relative } \\
\text { Frequency }\end{array}$ \\
\hline 46 & Aristida purpurea & 2.25 & 0.08 & 25 & 6.66 \\
\hline 47 & Mentha longifolia & 2.75 & 0.12 & 25 & 6.66 \\
\hline 48 & Cannabis sativa & 3 & 0.11 & 25 & 6.66 \\
\hline 49 & Conyza canadensis & 4.75 & 0.17 & 50 & 13.33 \\
\hline 50 & $\begin{array}{l}\text { Dichanthium } \\
\text { annulatum }\end{array}$ & 1.5 & 0.05 & 25 & 6.66 \\
\hline 51 & Digitaria ciliaris & 1.25 & 0.04 & 25 & 6.66 \\
\hline 52 & Eragrostis curvula & 4.25 & 0.15 & 50 & 13.33 \\
\hline 53 & $\begin{array}{l}\text { Parthenium } \\
\text { hysterophorus }\end{array}$ & 6 & 0.22 & 75 & 20 \\
\hline 54 & Themeda anathera & 1 & 0.009 & 25 & 6.66 \\
\hline \multirow[t]{2}{*}{55} & Tagetes minuta & 3 & 0.02 & 50 & 13.33 \\
\hline & Mean士S.E & $3 \pm 0.51$ & $0.1 \pm 0.02$ & $37.5 \pm 5.6$ & $10 \pm 1.5$ \\
\hline
\end{tabular}

Table 9. Density, frequency and IVI of Trees species at Havelian site in district Abbottabad.

\begin{tabular}{|c|c|c|c|c|c|c|c|}
\hline $\begin{array}{l}\text { Sr. } \\
\text { No }\end{array}$ & Scientific Name & $\begin{array}{c}\text { Density/ } \\
10 \mathrm{~m}^{2}\end{array}$ & $\begin{array}{l}\text { Relative } \\
\text { Density }\end{array}$ & Frequency & $\begin{array}{c}\text { Relative } \\
\text { Frequency }\end{array}$ & $\begin{array}{c}\text { Relative } \\
\text { Dominance }\end{array}$ & IVI \\
\hline 56 & Acacia modesta & 0.17 & 0.16 & 33.33 & 7.69 & 33.33 & 41.18 \\
\hline 57 & Ailanthus altissima & 0.07 & 0.06 & 33.33 & 7.69 & 33.33 & 41.08 \\
\hline 58 & Albizia procera (Roxb) Benth & 0.02 & 0.02 & 33.33 & 7.69 & 33.33 & 41.04 \\
\hline 59 & Broussonetia papyrifera & 0.27 & 0.25 & 66.66 & 15.38 & 66.66 & 82.29 \\
\hline 60 & $\begin{array}{c}\text { Eucalyptus camaldulensis } \\
\text { Dalbergia sissoo }\end{array}$ & 0.05 & 0.04 & 33.33 & 7.69 & 33.33 & 41.06 \\
\hline 61 & Ficus palmate & 0.15 & 0.13 & 66.66 & 15.38 & 66.66 & 82.17 \\
\hline 62 & Melia azedarach & 0.12 & 0.11 & 66.66 & 15.38 & 66.66 & 82.15 \\
\hline 63 & Olea ferruginea Royle & 0.02 & 0.02 & 33.33 & 7.69 & 33.33 & 41.04 \\
\hline 64 & Robinia pseudoacacia & 0.1 & 0.09 & 33.33 & 7.69 & 33.33 & 41.11 \\
\hline \multirow[t]{2}{*}{65} & & 0.07 & 0.06 & 33.33 & 7.69 & 33.33 & 41.08 \\
\hline & Mean士S.E & $0.1 \pm 0.02$ & $0.1 \pm 0.02$ & $43.3 \pm 5.1$ & $10 \pm 1.2$ & $43.3 \pm 5.1$ & $53.4 \pm 6.3$ \\
\hline
\end{tabular}


Table 10. Density and frequency of Shrubs species at Havelian site in district Abbottabad.

\begin{tabular}{cccccc}
\hline Sr. No & Scientific Name & Density $/ \mathbf{5} \mathbf{~}^{\mathbf{2}}$ & Relative Density & Frequency & Relative Frequency \\
\hline $\mathbf{6 6}$ & Dicliptera clinopodia & 0.46 & 0.15 & 33.33 & 14.28 \\
$\mathbf{6 7}$ & Malvastrum coromandelianum & 2.06 & 0.67 & 66.66 & 28.57 \\
$\mathbf{6 8}$ & Punica granatum & 0.13 & 0.04 & 33.33 & 14.28 \\
$\mathbf{6 9}$ & Rumex hastatus & 0.33 & 0.1 & 66.66 & 28.57 \\
$\mathbf{7 0}$ & Ziziphus lotus & 0.06 & 0.02 & 33.33 & 14.28 \\
& Mean士S.E & $\mathbf{0 . 6 \pm 0 . 3 7}$ & $\mathbf{0 . 2 \pm 0 . 1 2}$ & $\mathbf{4 6 . 7 \pm 8 . 2}$ & $\mathbf{2 0 \pm 3 . 5}$ \\
\hline
\end{tabular}

Table 11. Density and frequency of Herbs species at Havelian site in district Abbottabad.

\begin{tabular}{|c|c|c|c|c|c|}
\hline Sr. No & Scientific Name & Density/ $\mathbf{m}^{2}$ & Relative Density & Frequency & Relative Frequency \\
\hline 71 & Achyranthes aspera lin & 0.33 & 0.006 & 33.33 & 6.24 \\
\hline 72 & Alternanthera pungens & 3 & 0.05 & 33.33 & 6.24 \\
\hline 73 & Cannabis sativa & 7.66 & 0.15 & 66.66 & 12.49 \\
\hline 74 & Chenopodium ambrosioides & 2 & 0.03 & 33.33 & 6.24 \\
\hline 75 & Cynodon dactylon & 9.66 & 0.18 & 66.66 & 12.49 \\
\hline 76 & Ipomoea purpurea & 1 & 0.01 & 33.33 & 6.24 \\
\hline 77 & Parthenium hysterophorus & 7 & 0.13 & 66.66 & 12.49 \\
\hline 78 & Pennisetum hohenackeri & 6 & 0.11 & 33.33 & 6.24 \\
\hline 79 & Oxalis corniculata & 7 & 0.13 & 66.66 & 12.49 \\
\hline \multirow[t]{2}{*}{80} & Rumex crispus & 7.33 & 0.14 & 100 & 18.75 \\
\hline & Mean \pm S.E & $5.1 \pm 1.02$ & $0.1 \pm 0.02$ & $53.3 \pm 7.4$ & $10 \pm 1.4$ \\
\hline
\end{tabular}

Table 12. Density, frequency and IVI of Trees species at Langra site in district Abbottabad.

\begin{tabular}{|c|c|c|c|c|c|c|c|}
\hline Sr. No & Scientific Name & $\begin{array}{c}\text { Density/ } 10 \\
\mathrm{~m}^{2}\end{array}$ & $\begin{array}{l}\text { Relative } \\
\text { Density }\end{array}$ & Frequency & $\begin{array}{c}\text { Relative } \\
\text { Frequency }\end{array}$ & $\begin{array}{c}\text { Relative } \\
\text { Dominance }\end{array}$ & IVI \\
\hline 81 & Broussonetia papyrifera & 0.7 & 0.56 & 66.66 & 22.22 & 66.66 & 89.44 \\
\hline 82 & Dalbergia sissoo & 0.16 & 0.13 & 66.66 & 22.22 & 66.66 & 89.01 \\
\hline 83 & $\begin{array}{c}\text { Eucalyptus } \\
\text { camaldulensis }\end{array}$ & 0.03 & 0.02 & 33.33 & 11.11 & 33.33 & 44.46 \\
\hline 84 & Ficus palmate & 0.23 & 0.18 & 66.66 & 22.22 & 66.66 & 89.06 \\
\hline 85 & Grewia optiva & 0.06 & 0.05 & 33.33 & 11.11 & 33.33 & 44.49 \\
\hline \multirow[t]{2}{*}{86} & Morus alba & 0.03 & 0.02 & 33.33 & 11.11 & 33.33 & 44.46 \\
\hline & Mean士S.E & $0.2 \pm 0.10$ & $0.2 \pm 0.08$ & $50 \pm 7.5$ & $16.7 \pm 2.5$ & $50 \pm 7.5$ & $66.8 \pm 10$ \\
\hline
\end{tabular}

Table 13. Density and frequency of Shrubs species at Langra site in district Abbottabad.

\begin{tabular}{|c|c|c|c|c|c|}
\hline Sr. No & Scientific Name & Density $/ 5 \mathrm{~m}^{2}$ & Relative Density & Frequency & $\begin{array}{l}\text { Relative } \\
\text { Frequency }\end{array}$ \\
\hline 87 & Dicliptera clinopodia & 0.2 & 0.06 & 33.33 & 19.99 \\
\hline 88 & Justicia adhatoda & 0.33 & 0.1 & 33.33 & 19.99 \\
\hline \multirow[t]{2}{*}{89} & Malvastrum coromandelianum & 2.66 & 0.83 & 100 & 60 \\
\hline & Mean士S.E & $1.1 \pm 0.80$ & $0.3 \pm 0.25$ & $55.6 \pm 22.2$ & $33.3 \pm 13.3$ \\
\hline
\end{tabular}


Table 14. Density and frequency of Herbs species at Langra site in district Abbottabad.

\begin{tabular}{cccccc}
\hline Sr. No & Scientific Name & Density $/ \mathbf{~ m}^{\mathbf{2}}$ & Relative Density & Frequency & Relative Frequency \\
\hline $\mathbf{9 0}$ & Achyranthes aspera linn & 1 & 0.025 & 33.33 & 6.25 \\
$\mathbf{9 1}$ & Amaranthus spinosus & 1 & 0.025 & 33.33 & 6.25 \\
$\mathbf{9 2}$ & Alternanthera pungens & 4.33 & 0.108 & 33.33 & 6.25 \\
$\mathbf{9 3}$ & Cannabis sativa & 2.66 & 0.066 & 66.66 & 12.5 \\
$\mathbf{9 4}$ & Chenopodium album & 0.66 & 0.016 & 33.33 & 6.25 \\
$\mathbf{9 5}$ & Cynodon dactylon & 7.5 & 0.225 & 66.66 & 12.5 \\
$\mathbf{9 6}$ & Dactyloctenium aegyptium & 4.66 & 0.116 & 33.33 & 6.25 \\
$\mathbf{9 7}$ & Eleusine indica & 3 & 0.075 & 33.33 & 6.25 \\
$\mathbf{9 8}$ & Oxalis corniculata & 6 & 0.15 & 66.66 & 12.5 \\
$\mathbf{9 9}$ & Parthenium hysterophorus & 3.33 & 0.083 & 66.66 & 12.5 \\
$\mathbf{1 0 0}$ & Rumex crispus & 4.33 & 0.108 & 66.66 & 12.5 \\
& Mean \pm S.E & $\mathbf{3 . 5 \pm 0 . 6 5}$ & $\mathbf{0 . 1 \pm 0 . 0 2}$ & $\mathbf{4 8 . 5} \mathbf{5 . 2}$ & $\mathbf{9 . 1} \mathbf{1 . 0}$ \\
\hline
\end{tabular}

Table 15. Density, frequency and IVI of Trees species at Jhangra site in district Abbottabad.

\begin{tabular}{cccccccc}
\hline Sr. No & Scientific Name & Density/ 10 $\mathbf{~ m}^{\mathbf{2}}$ & $\begin{array}{c}\text { Relative } \\
\text { Density }\end{array}$ & Frequency & $\begin{array}{c}\text { Relative } \\
\text { Frequency }\end{array}$ & $\begin{array}{c}\text { Relative } \\
\text { Dominance }\end{array}$ & IVI \\
\hline 101 & Acacia modesta & 0.17 & 0.1 & 75 & 15 & 75 & 90.1 \\
102 & Ailanthus altissima & 0.07 & 0.04 & 50 & 10 & 50 & 60.04 \\
103 & Broussonetia papyrifera & 0.57 & 0.34 & 75 & 15 & 75 & 90.34 \\
104 & Dalbergia sissoo & 0.12 & 0.07 & 25 & 5 & 25 & 30.07 \\
105 & Ficus palmata & 0.07 & 0.04 & 25 & 5 & 25 & 30.04 \\
106 & Grewia optiva & 0.07 & 0.04 & 50 & 10 & 50 & 60.04 \\
107 & Melia azedarach & 0.1 & 0.05 & 50 & 10 & 50 & 60.05 \\
108 & Morus alba & 0.05 & 0.03 & 50 & 10 & 50 & 60.03 \\
109 & Olea ferruginea Royle & 0.27 & 0.16 & 75 & 15 & 75 & 90.16 \\
110 & Zanthoxylum armatum & 0.15 & 0.08 & 25 & 5 & 25 & 30.08 \\
& Mean \pm S.E & $0.2 \pm 0.05$ & $0.1 \pm 0.03$ & $50 \pm 6.45$ & $10 \pm 1.29$ & $50 \pm 6.45$ & $60.1 \pm 7.7$
\end{tabular}

Table 16. Density and frequency of Shrubs species at Jhangra site in district Abbottabad.

\begin{tabular}{cccccc}
\hline Sr. No & Scientific Name & Density/ $\mathbf{5} \mathbf{~ m}^{\mathbf{2}}$ & Relative Density & Frequency & $\begin{array}{c}\text { Relative } \\
\text { Frequency }\end{array}$ \\
\hline $\mathbf{1 1 1}$ & Barleria cristata & 0.55 & 0.11 & 50 & 22.22 \\
$\mathbf{1 1 2}$ & Calotropis gigantean & 0.15 & 0.03 & 25 & 11.11 \\
$\mathbf{1 1 3}$ & Dicliptera clinopodia & 0.5 & 0.1 & 25 & 11.11 \\
$\mathbf{1 1 4}$ & Elaeagnus umbellate & 0.1 & 0.02 & 25 & 11.11 \\
$\mathbf{1 1 5}$ & Justicia adhatoda & 0.85 & 0.18 & 25 & 11.11 \\
$\mathbf{1 1 6}$ & Malvastrum coromandelianum & 2.55 & 0.54 & 75 & 33.33 \\
& Mean \pm S.E & $\mathbf{0 . 8 \pm 0 . 3 7}$ & $\mathbf{0 . 2 \pm 0 . 0 8}$ & $\mathbf{3 7 . 5} \pm \mathbf{8 . 5}$ & $\mathbf{1 6 . 7 \pm 3 . 8}$ \\
\hline
\end{tabular}

Table 17. Density and frequency of Herbs species at Jhangra site in district Abbottabad.

\begin{tabular}{|c|c|c|c|c|c|}
\hline Sr. No & Scientific Name & Density/ $\mathbf{m}^{2}$ & Relative Density & Frequency & Relative Frequency \\
\hline 117 & Cannabis sativa & 3.5 & 0.09 & 50 & 13.33 \\
\hline 118 & Eragrostis curvula & 4.5 & 0.12 & 25 & 6.66 \\
\hline 119 & Euphorbia hirta & 1.25 & 0.03 & 25 & 6.66 \\
\hline 120 & Dichanthium annulatum & 9.25 & 0.26 & 50 & 13.33 \\
\hline 121 & Oxalis corniculata & 8.75 & 0.24 & 75 & 20 \\
\hline 122 & Parthenium hysterophorus & 5.25 & 0.14 & 75 & 20 \\
\hline 124 & Rhynchosia minima & 0.25 & 0.007 & 25 & 6.66 \\
\hline \multirow[t]{2}{*}{125} & Rumex crispus & 2.5 & 0.07 & 50 & 13.33 \\
\hline & Mean \pm S.E & $4.4 \pm 1.16$ & $0.1 \pm 0.03$ & $46.9 \pm 7.4$ & $12.5 \pm 2.0$ \\
\hline
\end{tabular}




\section{Discussion}

The previous published literature shows that D. vagabunda is commonly found abundant in vegetation in sub-tropical forest and scrub areas in India as mentioned by Thakur et al. (2010), whereas Jayson and Mathew (2000) reported its abundance found in tropical evergreen forest in Kerala. Moreover, Mirza and Wasiq (2012) revealed habitat utilization of $D$. vagabunda in cultivated trees and scrub forest areas, not in desert without trees.

The present study investigated the population density of D. vagabunda from October 2018 to June 2019 at five study sites of the district Abbottabad. Our study agrees with Bashir et al. (2012), who reported the estimated population density of $D$. vagabunda $(0.045 \pm 0.015)$ during bank searches of avian species in upper Ganges in India. Moreover, Palita et al. (2011) documented population density abundance of $D$. vagabunda $(0.73 \pm 0.96)$ at Morus alba, $(0.53 \pm 0.35)$ in Ficus palmata, $(0.2 \pm 0.41)$ in Buddleja asiatica, $(0.13 \pm 0.35)$ in Phacelia crenulata, $(0.26 \pm 0.45)$ in Rubus ellipticus and $(0.9 \pm 0.99)$ in Pyrus communis on fruit plants in summer fruiting season in Uttarakhand in India.

Similar results were reported earlier on by Saikia et al. (2014) for D. vagabunda 0.57 in dry season and 0.43 in wet season in North-East India, because the area is large as compared to our study area. However, Awan et al. (2009) also reported relative abundance of 0.010 D. vagabunda in Azad Jammu and Kashmir, Pakistan.

Chatterjee et al. (2014) reported density of $D$. vagabunda in Grasslands (0.00/ha), Swamp forests (0.00/ha), Riverine forests (1.04/ha), Miscellaneous plantations (1.56/ha), Forest edges (2.60/ha) and Wetlands (0.00/ha) in west Bengal India, because the area is large as compared to our study area. Whereas, Roy et al. (2012) reported the estimated population density of $D$. vagabunda in Gorumara National Park (4.9/ha), in Buxa Tiger Reserve (0.0/ha), and in Rasik Beel Wetland Complex (3.9/ha) in three different regions of North Bengal in India, because the area is too large as compared to our study area.

The present study is supported by Trivedi (2006), who reported the population density of $D$. vagabunda $(1.93 \pm 0.86)$ in Gujarat in India. Similar results to the present study were obtained by Sidhu et al. (2010), where they also described average relative abundance of $D$. vagabunda in Forest (1.00), in Buffer (4.56) and in village (3.75) in Thattekad and in Forest (0.00), in Buffer (0.00) and in village (0.00) in Anamalai Hills in the Southern Western Ghats in India, because the area is large as compared to our study area.

No significant difference ( $p>0.05$ ) was found among monthly $D$. vagabunda population densities/ha in hilly and plane areas through Analysis of Variance (ANOVA) (Table 1). However, Statistical analysis (t-test) indicates a significant difference $(p<0.05)$ among population densities/ha between morning and evening time population.

\section{Conclusion}

The results of current study explain the wide distribution of Rufous treepie in District Abbottabad. At all selected sites the specie was not affected by the human population disturbance of nearby areas during study period, most probably because this bird belongs to family corvidae and is not hunted by locals, hence population is stable. Although habitat destruction is a major threat to population decline, however variety of vegetation is available for this bird species at all selected study sites, and as a result it prefers dominant IVI of trees $66.8 \pm 10$ having population density $0.11 \pm 0.033$ at Langra, whereas, minimum IVI of trees $50.1 \pm 6.9$ with population density $0.13 \pm 0.029$ at Bakot. Our results confirm that all selected hilly and plane study sites of Abbottabad were suitable habitat for D. vagabunda.

\section{References}

ALI, A., BADSHAH, L. and HUSSAIN, F., 2018. Vegetation structure and threats to Montane Temperate ecosystems in Hindukush Range, Swat, Pakistan. Applied Ecology and Environmental Research, vol. 16, no. 4, pp. 4789-4811. http://dx.doi.org/10.15666/ aeer/1604_47894811.

ALI, S., RAHMAN, Z., KHAN, S., KHAN, T., ALI, W. and IQBAL, A., 2017. Assessment of land use pattern in district Abbottabad through geographic information system and remote sensing. Biologia, vol. 63, no. 1, pp. 87-96.

ARYAL, A., RAUBENHEIMER, D., SUBEDI, S. and KATTEL, B., 2010. Spatial Habitat Overlap and Habitat Preference of Himalayan Musk Deer (" Moschus chrysogaster") in Sagarmatha (Mt. Everest) National Park, Nepal. Current Research Journal of Biological Sciences, vol. 2, no. 3, pp. 217-225.

AWAN, M.N., RAFIQUE, S.M. and CHAUDRY, M.I., 2009. Changes to the checklist of the birds of Muzaffarabad city, Azad Jammu and Kashmir, Pakistan. Podoces, vol. 4, no. 2, pp. 117-123.

BASHIR, T., BEHERA, S.K., KHAN, A. and GAUTAM, P., 2012. An inventory of mammals, birds and reptiles along a section of the river and banks of upper Ganges, India. Journal of Threatened Taxa, vol. 4, no. 9, pp. 2900-2910. http://dx.doi.org/10.11609/ JoTT.02692.2900-10.

BIRDLIFE INTERNATIONAL, 2013. Species fact sheet: Dendrocitta vagabunda. Cambridge.

BUCKLAND, S.T., ANDERSON, D.R., BURNHAM, K.P., LAAKE, J.L., BORCHERS, D.L. and THOMAS, L., 2001. Introduction to distance sampling: estimating abundance of biological populations. Oxford: Oxford University Press.

BUCKLAND, S.T., MARSDEN, S.J. and GREEN, R.E., 2008. Estimating bird abundance: making methods work. Bird Conservation International, vol. 18, no. S1, pp. S91-S108. http://dx.doi. org/10.1017/S0959270908000294.

CHATTERJEE, A., ADHIKARI, S., BARIK, A. and MUKHOPADHYAY, S.K., 2014. The mid-winter assemblage and diversity of bird populations at Patlakhawa Protected Forest, Coochbehar, West Bengal, India. The Ring, vol. 35, no. 1, pp. 31-53. http://dx.doi. org/10.2478/ring-2013-0002.

GRIMMETT, R., ROBERTS, T.J., INSKIPP, T. and BYERS, C., 2008. Birds of Pakistan. London: A\&C Black.

HORNMAN, M., 2007. Northern E' central Pakistan. Surfbirds.

IUCN PAKISTAN, 2004. Abbottabad: state of the environment and development. Karachi, Pakistan: IUCN Pakistan and NWFP, 136 p.

JADOON, A., BIBI, S. and REHMAN, A., 2019. Birds population in district Haripur, Khyber Pakhtunkhwa, Pakistan. Journal of Wildife and Ecology, vol. 3, no. 3, pp. 18-25.

JAYSON, E.A. and MATHEW, D.N., 2000. Diversity and speciesabundance distribution of birds in the tropical forests of Silent 
Valley, Kerala. Journal of the Bombay Natural History Society, vol. 97, no. 3, pp. 390-399.

JOSHI, K.K., BHATT, D. and THAPLIYAL, A., 2012. Avian diversity and its association with vegetation structure in different elevational zones of Nainital district (Western Himalayan) of Uttarakhand. International Journal of Biodeversity and Conservation, vol. 4, no. 11, pp. 364-370. http://dx.doi.org/10.5897/IJBC11.243.

MIRZA, Z. B. and WASIQ H., 2012. Handbook of birds of Pakistan: a field guide. Pakistan: WWF Pakistan for the Conservation of Birds, 305 p.

PALITA, S.K., PONKSHE, A.V. and DHAR, U., 2011. Habitat enrichment and its impact on avian diversity: a study at GBPIHED, KosiKatarmal, Uttarakhand, India. Current Science, vol. 100, no. 11, pp. 1681-1689

RALPH, C.J., SAUER, J.R. and DROEGE, S., 1995. Monitoring bird populations by point counts. Albany: U.S. Department of Agriculture, $191 \mathrm{p}$.

ROLANDO, A., CAPRIO, E., RINALDI, E. and ELLENA, I., 2007. The impact of high-altitude ski-runs on alpine grassland bird communities. Journal of Applied Ecology, vol. 44, no. 1, pp. 210-219. http://dx.doi.org/10.1111/j.1365-2664.2006.01253.x.

ROY, U.S., BANERJEE, P. and MUKHOPADHYAY, S.K., 2012. Study on avifaunal diversity from three different regions of North Bengal, India. Asian Journal of Conservation Biology, vol. 1, no. 2, pp. 120-129.

SAIKIA, M.K., SAIKIA, P.K. and BHATTA, R., 2014. Management perspectives for avian population conservation and enrichmentn in deepor beel Ramsar site, North-East India. Journal of Global Biosciences, vol. 3, no. 2, pp. 428-451.

SEKERCIOGLU, C.H., 2006. Increasing awareness of avian ecological function. Trends in Ecology E Evolution, vol. 21, no. 8, pp. 464-471. http://dx.doi.org/10.1016/j.tree.2006.05.007. PMid:16762448.
SHAHABUDDIN, G., KUMAR, R. and VERMA, A., 2006. Annotated checklist of the birds of Sariska Tiger Reserve, Rajasthan, India. Indian Birds, vol. 2, no. 3, pp. 71-76.

SIDHU, S., SHANKAR RAMAN, T.R. and GOODALE, E., 2010. Effects of plantations and home-gardens on tropical forest bird communities and mixed-species bird flocks in the southern Western Ghats. Journal of the Bombay Natural History Society, vol. 107, no. 2, pp. 91.

SIGDEL, S.R., 2008. Altitudinally coordinated pattern of plant community structure in the Shivapuri National Park, Nepal. Banko Janakari, vol. 18, no. 1, pp. 11-17. http://dx.doi.org/10.3126/ banko.v18i1.2161.

SINGH, R., KOUR, D.N., AHMAD, F. and SAHI, D.N., 2013. Species diversity, relative abundance and habitat use of the bird communities of Tehsil Chenani, District Udhampur, Jammu and Kashmir, India. Indian Journal of Life Sciences, vol. 2, no. 2, pp. 81.

STEPHENS, P.A., VIEIRA, M.V., WILLIS, S.G. and CARBONE, C., 2019. The limits to population density in birds and mammals. Ecology Letters, vol. 22, no. 4, pp. 654-663. http://dx.doi.org/10.1111/ ele.13227. PMid:30724435.

THAKUR, M.L., MATTU, V.K., MATTU, N., SHARMA, V.N., BHARDWAJ, R. and THAKUR, V., 2010. Bird Diversity in Sarkaghat Valley, Mandi (Himachal Pradesh), India. Asian Journal of Experimental Biological Sciences, vol. 1, no. 4, pp. 940-950.

TRIVEDI, P.G., 2006. Ecology and conservation of avifauna of some forested areas in Gujarat, India. Rajkot: Saurashtra University. Doctoral dissertation in Philosophy in Wildlife Science.

ULLAH, Z., ULLAH, I., ULLAH, I., MAHMOOD, S. and IQBAL, Z., 2020. Poaching of Asiatic black bear: evidence from Siran and Kaghan valleys, Pakistan. Global Ecology and Conservation, vol. 24, pp. e01351. http://dx.doi.org/10.1016/j.gecco.2020.e01351. 